\title{
THE TRANSITIVE GROUPS OF DEGREE 48 AND SOME APPLICATIONS
}

\author{
DEREK HOLT, GORDON ROYLE, AND GARETH TRACEY
}

\begin{abstract}
The primary purpose of this paper is to report on the successful enumeration in MAGMA of representatives of the 195826352 conjugacy classes of transitive subgroups of the symmetric group $S_{48}$ of degree 48. In addition, we have determined that 25707 of these groups are minimal transitive and that 713 of them are elusive. The minimal transitive examples have been used to enumerate the vertex-transitive graphs of degree 48, of which there are 1538868366 , all but $0.1625 \%$ of which arise as Cayley graphs. We have also found that the largest number of elements required to generate any of these groups is 10, and we have used this fact to improve previous general bounds of the third author on the number of elements required to generate an arbitrary transitive permutation group of a given degree. The details of the proof of this improved bound will be published by the third author as a separate paper.
\end{abstract}

\section{INTRODUCTION}

Since late in the 19th century, significant effort has been devoted to compiling catalogues and databases of various types of groups, including complete lists of (representatives of the conjugacy classes of) the transitive and primitive subgroups of the symmetric groups of small degree. For the transitive groups, earlier references include [24, 23] (with corrections in [25]) for degrees up to 12, [12] for degrees up to 31, 2] for the significantly more difficult case of degree 32, and [11] for degrees 33-47. (There are 2801324 groups of degree 32, and a total of 501045 groups of all other degrees up to 47.) Apart from the early work of Miller, these lists have been compiled by computer, using GAP and MAGMA.

Degree 48 is once again significantly more difficult than earlier degrees, because there are many more groups and the computations involved need more time and computer memory. The main purpose of this paper is to report on the successful enumeration of conjugacy class representatives of the transitive subgroups of degree 48, which is the topic of Section 2, There are a total of 195826352 of these subgroups. The computations were carried out in MAGMA and required a total of about one year of cpu-time. The complete list of these subgroups is available in MAGMA using an optional database that can be downloaded by users from the MAGMA website. Although we carried out these computations serially on a single processor and, due to various logistical problems, they took more than two years of real time to complete, they are intrisically extremely parallelisable: about $98 \%$ of the cpu-time was for imprimitive groups with blocks of size 2 and, as we shall explain shortly, that case splits into 25000 independent calculations.

We anticipate that it would be feasible to extend the catalogues up to degree 63 , but that degree 64 will remain out of range for the foreseeable future.

2010 Mathematics Subject Classification. 20B40, 20B35, 05C30.

Key words and phrases. transitive group; vertex-transitive graph; census; catalogue; generator number. 


\begin{tabular}{|r|rrr|}
\hline MinBlockSize & TransGps & MinTrans & Elusive \\
\hline 2 & 192327620 & 15046 & 108 \\
3 & 3397563 & 10625 & 590 \\
4 & 94121 & 36 & 3 \\
6 & 5011 & 0 & 12 \\
8 & 1275 & 0 & 0 \\
12 & 103 & 0 & 0 \\
16 & 646 & 0 & 0 \\
24 & 9 & 0 & 0 \\
Primitive & 4 & 0 & 0 \\
\hline Total & 195826352 & 25707 & 713 \\
\hline
\end{tabular}

TABLE 1. Numbers of transitive, minimal transitive, and elusive groups of degree 48

We subsequently used our catalogue to identify those groups of degree 48 that are minimal transitive (that is, they have no proper transitive subgroups) and those that are elusive (that is, they contain no fixed-point-free elements of prime order). We shall report on this in Section 3 . The various counts of groups involved are summarised in Table 1, where the imprimitive groups have been counted according to the smallest size of a block of imprimitivity.

In Section 4, we describe the computation of the vertex-transitive graphs of order 48, along with some associated data.

We denote the smallest size of a generating set of a group $G$ by $d(G)$. We have established by routine computations, that $d(G) \leqslant 10$ for all transitive groups of degree 48 . In fact, the only examples with $d(G)=10$ have minimal block size 3 and have 10-generator transitive groups of degree 32 as quotients. The groups with block size 2 all satisfy $d(G) \leqslant 9$.

As we shall explain in Section 5, these bounds on $d(G)$ have enabled the third author to remove the exceptional cases of the general bound on $d(G)$ for transitive permutation group of degree $n$ that he established in [28, and thereby to complete the proof of the following result, where logarithms are to the base 2 .

Theorem 1.1. Let $G$ be a transitive permutation group of degree $n$. Then

$$
d(G) \leqslant\left\lfloor\frac{c n}{\sqrt{\log n}}\right\rfloor
$$

where $c:=\frac{\sqrt{3}}{2}$.

(It was proved by Lucchini in [19] that this result holds for some unspecified constant $c$.) Since the proof of this result involves some lengthy case-by-case analyses, we shall just summarise it in Section 5 of this paper, and the details will be published separately by the third author [29].

In a related application, the third author is now able to improve a previously unpublished result bounding the constant $d$ in the result proved in [20] that $d(G) \leqslant d \log n / \sqrt{\log \log n}$ for primitive subgroups $G$ of $S_{n}$. 
Notation: For a finite group $G$, we will write $\Phi(G), R(G),[G, G]$ for the Frattini subgroup, soluble radical, and derived subgroup of $G$, respectively. We will mostly use the notation from [31] for group names, although we simply write $n$ for the cyclic group of order $n$ when there is no danger of confusion.

\section{Computing the transitive groups of Degree 48}

The primitive permutation groups are known up to degree 40951 (see [4]) and are incorporated into the databases of both MAGMA and GAP, and so we need only consider the imprimitive groups. By definition, if a group $G$ acting transitively on the set $\Omega$ of size $n$ is not primitive, then there is at least one partition of $\Omega$ into a block system $\mathcal{B}$ such that $G$ permutes the blocks of $\mathcal{B}$. If we let $G^{\mathcal{B}}$ denote the action of $G$ on the blocks ("the top group") and if $\mathcal{B}$ has $n / k$ blocks of size $k$, then $G^{\mathcal{B}}$ is a transitive permutation group of degree $n / k$. We say that the block system $\mathcal{B}$ is minimal if $k$ is minimal among block systems with $k>1$. Then we can associate to each group $G$ a set of pairs of the form

$$
\left\{\left(k, G^{\mathcal{B}}\right): \mathcal{B} \text { is a minimal block system for } G \text { with blocks of size } k\right\} .
$$

If this set contains more than one pair (imprimitive groups may of course have more than one minimal block system), then we wish to distinguish just one of them. Thus we define the signature of an imprimitive permutation group to be the lexicographically least pair $\left(k, G^{\mathcal{B}}\right)$ associated with $G$, where the second component is indexed according to its order in the list of transitive groups of degree $n / k$ already in MAGmA. But note that it can happen that two different minimal block systems of $G$ define the same signature.

We separate the computation into parts, with each part constructing only the groups with a particular signature. Given an integer $k$ such that $1<k<n$, and a transitive group $H$ of degree $n / k$, the wreath product $S_{k} \prec H$ contains (a conjugate of) every transitive group of degree $n$ with signature $(k, H)$. So these groups can all be found by exploring the subgroup lattice of $S_{k} \prec H$ (although there are complications arising from the fact that we want representatives of subgroups up to conjugacy in $S_{n}$.)

A naive approach to the problem for a fixed $k$, is to deal with all candidates $H$ simultaneously, by starting with $S_{k} \prec S_{n / k}$, and repeatedly using the MaximalSubgroups command of MAGMA, thereby traversing the subgroup lattice downwards and in a breadth-first fashion, pruning each branch of the search as soon as it produces groups with signature differing from $H$, while using conjugacy tests to avoid duplication. (We also have to eliminate duplicates arising from a group preserving more than one minimal block system with blocks of size $k$.) This was successfully applied in all cases to the transitive groups of degrees $33-47$, and we refer the reader to [11, Section 2] for further details. In degree 48, we successfully applied this method to groups with signatures $(k, H)$ with $k \geqslant 6$; that is for $k=6,8,12,16$ and 24 . The cpu-times in these cases were of order 10 hours, 30 minutes, 3 minutes, 70 minutes, and a few seconds, respectively.

For the examples with $k=2,3$ and 4, we used the methods described in [11, Section 3] (and also in [2, Section 2.2] for $k=2$ ).

\footnotetext{
${ }^{1}$ This has recently been extended to degree 8191 by Ben Stratford, a student of the first author.
} 
The methods for $k=3$ were essentially the same as in degree 36 , but considerably more time-consuming. We have $G \leqslant S_{3} \prec S_{16} \cong C_{3} \prec\left(C_{2} \prec S_{16}\right)$. Let $\rho$ be the induced projection of $G$ onto $C_{2} \prec S_{16}$. Then, since we are assuming that $G$ is transitive, $\rho(G)$ must project onto a transitive subgroup of $S_{16}$, and the existing catalogues contain the 1954 possibilities for this projection. Furthermore, either

(i) $\rho(G)$ is a transitive subgroup of $S_{32}$, in which case we can use the existing catalogue 2801324 as a list of candidates for $\rho(G)$; or

(ii) $\rho(G)$ is an intransitive groups of degree 32 that projects onto a transitive subgroup of $S_{16}$. In that case, it is not hard to show that $G$ must be conjugate to the natural complement of the base group of $C_{2} \imath H$, where $H$ is one of the 1954 transitive groups of degree 16. (We also checked this computationally.)

We enumerated the groups with $k=3$ by considering each of the $2801324+1954$ possibilities for $\rho(G)$ in turn. This involves a cohomology computation, which is analogous to that for the case $k=2$, which we shall discuss below. The computation for $k=3$ took a total of about 104 hours of cpu-time. Of the 3397563 groups on this list, $\rho(G)$ is transitive for all except 55715 . Similarly, for $k=4$, we used the same techniques as in degrees 36 and 40, and the total cpu-time was about 9 hours.

The vast majority of the computational work was for the case $k=2$, and we shall briefly recall how we proceed in this case. We have $G \leqslant W:=C_{2}$ 乙 $H$, where $H:=G^{\mathcal{B}}$ is one of the groups in the known list of 25000 transitive groups of degree 24. Again we calculate those groups with signature $(2, H)$ for each individual group $H$, and the 25000 calculations involved are independent and could in principal be done in parallel.

Let $K \cong C_{2}^{24}$ be the kernel of the action of $W$ on $\mathcal{B}$. Then we can regard $K$ as a module for $H$ over the field $\mathbb{F}_{2}$ of order 2 , and $M:=G \cap K$ is an $\mathbb{F}_{2} H$-submodule. We can use the Magma commands GModule and Submodules to find all such submodules. In fact, since we are looking for representatives of the conjugacy classes of transitive subgroups of $W$, we only want one representative of the conjugation action of $N:=C_{2} 2 N_{S_{24}}(H)$ on the set all $\mathbb{F}_{2} H$-submodules $M$ of $K$, and we use the MAGMA command IsConjugate to find such representatives.

Now, for each such pair $(H, M)$, the transitive groups $G$ with $H=G^{\mathcal{B}}$ and $M=G \cap K$ correspond to complements of $K / M$ in $H / M$, and the $H$-conjugacy classes of such complements correspond to elements of the cohomology group $H^{1}(H, K / M)$, which can be computed in MAGMA.

We also need to test these groups $G$ for conjugacy under the action of $N_{N}(M)$. In some cases when $H^{1}(H, K / M)$ is reasonably small, this can be done in straightforward fashion using MAGMA's IsConjugate function. But in many cases this was not feasible, and we had to use the method using an induced action on the cohomology group that is described in detail in [2, Section 2.2]. Finally, for each $G$ that we find, we need to find all block systems with block size 2 preserved by $G$, so that we can eliminate occurrences of groups that are conjugate in $S_{n}$ but arise either for distinct pairs $(H, M)$ or more than once for the same pair. Again we refer the reader to [2, Section 2.2] for further details.

Here are some statistical details concerning some of these calculations. 
- The numbers of groups arising from the 25000 candidates for the top group $H$ ranges from 3 to 3642186 , with average 7693 and median 778. This number is less than 10000 for more than $90 \%$ of the top groups. For the majority of these groups $H$, the computations were fast. For examples, for the groups $H=\operatorname{TransitiveGroup}(24, k)$ with $20000<k \leqslant 25000,(20 \%$ of the groups $H$ ) the total cpu-time was about 92.4 hours (just over $1 \%$ of the total) and the total number of groups $G$ that arise is 2963853 (about $1.5 \%$ of the total).

- The highest dimension of a cohomology group $H^{1}(H, M)$ was 26 . In that case, $\left|H^{1}(H, M)\right|=$ $2^{26}=67108864$, where elements of $H^{1}(H, M)$ are represented by 26 binary digits. This is important because of the orbit computation on the elements of $H^{1}(H, M)$. If an example of much higher dimension than this had been encountered (which we might expect to be the case for a corresponding attempt to find the transitive groups of degree 64 ), then this orbit computation might not have been feasible. This occurred with $H=$ TransitiveGroup $(24,4010)$ and $|M|=2^{12}$, and the pair $(H, M)$ gave rise to 201792 groups $G$.

- The case $H=$ TransitiveGroup $(24,11363)$ resulted in the largest number of groups $G$, namely 3642186 . There were 240 possibilities for $M$. This case took about 34 hours of cpu-time, using about 73GBytes RAM.

- The pair $(H, M)$ that resulted in the most groups $G$, namely 1054720 , arose with $H=$ TransitiveGroup $(24,13329)$ and $|M|=2^{7}$. Although $H^{1}(H, M)$ had dimension only 22 in this case, there were many more orbits of the action than in the case with dimension 26 discussed above.

\section{Minimal transitive AND Elusive Groups}

3.1. Minimal transitive groups. For many applications that involve considering all possible transitive actions of a certain degree, it is sufficient to consider only the minimal transitive groups i.e., transitive groups with no proper transitive subgroups. (One example of this was discussed in [11, Section 5], where all vertex-transitive graphs of degrees 33-47 are constructed.) Testing if a transitive group is minimal can be done by finding all of its maximal subgroups and verifying that none are transitive. As most of the groups are not minimal transitive, it proves useful in practice to first construct some random subgroups in an attempt to find a transitive proper subgroup, only undertaking the more expensive step of finding all maximal subgroups if this fails.

There are a total of 25707 minimal transitive groups, all of which have minimal blocks of sizes 2,3 or 4 - the exact numbers of minimal transitive groups with smallest blocks of each size are given in Table 1 .

3.2. Elusive groups. One of the major reasons to construct catalogues of combinatorial objects is to gather evidence relating to conjectures or other open questions. Even if a newly-constructed catalogue does not directly contain a counterexample to a conjecture (thereby immediately resolving it), it can be useful in refining a researcher's intuition regarding both the typical and extremal objects in the catalogue. 
A permutation group $G$ is called elusive if it contains no fixed-point-free elements (i.e., derangements) of prime order. Elusive groups are interesting because of their connection to Marušič's Polycirculant Conjecture [22] which asserts that the automorphism group of a vertextransitive digraph is never elusive. In principle, a positive resolution of the polycirculant conjecture may simplify the construction and analysis of vertex-transitive graphs and digraphs, as it would then be possible to assume the presence of an automorphism with $n / p$ cycles of length $p$ for some prime $p$. Early catalogues of vertex-transitive graphs often used ad hoc arguments to show that all transitive groups of the specific degrees under consideration have a suitable derangement of prime order.

A permutation group $G$ is called 2-closed if there is no group properly containing $G$ with the same orbitals as $G$. The automorphism group of a vertex-transitive digraph is necessarily 2-closed, because it is already the maximal group (by inclusion) that fixes the set of arcs of the digraph, which is a union of some of the orbitals. The conjecture can thus be strengthened to the assertion that there are no elusive 2-closed transitive groups, as proposed by Klin and Marušič at the 15th British Combinatorial Conference [16].

One might hope that there are simply no elusive groups at all, in which case both conjectures would hold vacuously, but in fact there are a number of sporadic examples of elusive groups and a handful of infinite families. However all the known elusive groups are not 2-closed, so do not provide counterexamples for either conjecture.

It is relatively easy to test the groups for the property of being elusive by checking to see if any of the conjugacy class representatives are derangements of prime order. For the larger groups, it is often faster to first generate some number of randomly selected elements inside each of the Sylow subgroups in the hope of stumbling on a suitable derangement without the cost of computing all the conjugacy classes.

The results of this computation reveal that there are 713 elusive groups of degree 48 , with orders ranging from 5184 to 806215680000 . The numbers of elusive groups of degree 48 with each minimal blocksize are given in Table 1, If an elusive group has minimal blocks of different sizes (say 2 and 3), then it is grouped and counted according the smaller of the sizes.

Of these groups 700 have a unique minimal normal subgroup, and while each of the remaining 13 groups has multiple minimal normal subgroups, these minimal normal subgroups are conjugate in $S_{48}$. Therefore we can partition the elusive groups according to the unique conjugacy class of their minimal normal subgroup(s).

Collectively, the 713 elusive groups share just 7 pairwise non-conjugate minimal normal subgroups. Table 2 shows the different minimal normal subgroups that occur and the number of elusive groups with that particular minimal normal subgroup. In addition, it gives the order of the normalizer (in $S_{48}$ ) of that subgroup, while the final column shows the possible minimal block sizes that occur for that minimal normal subgroup. All but one of the possible minimal normal subgroups are elementary abelian, but two non-conjugate (but obviously isomorphic) groups of orders $2^{8}$ and $3^{8}$ occur. For example, the first row shows that an elusive group with minimal normal subgroup $C_{3}^{4}$ either has minimal blocks of size 2 (only) or minimal blocks of sizes both 2 and 3 . 


\begin{tabular}{lccc}
\hline Group & $\mid$ Normalizer $\mid$ & Frequency & Min. Blocks \\
\hline$C_{3}^{4}$ & $2^{17} \cdot 3^{18}$ & 22 & $\{2\}$ or $\{2,3\}$ \\
$C_{2}^{8}$ & $2^{28} \cdot 3^{3}$ & 3 & $\{4\}$ \\
$C_{2}^{8}$ & $2^{43} \cdot 3^{5}$ & 75 & $\{2\}$ \\
$C_{3}^{8}$ & $2^{19} \cdot 3^{21}$ & 575 & $\{3\}$ \\
$C_{3}^{8}$ & $2^{11} \cdot 3^{17} \cdot 7$ & 2 & $\{3\}$ \\
$C_{2}^{16}$ & $2^{39} \cdot 3^{10} \cdot 5 \cdot 7$ & 24 & $\{2\}$ \\
$\mathrm{A}(6)^{4}$ & $2^{23} \cdot 3^{9} \cdot 5^{4}$ & 12 & $\{6\}$ \\
\hline
\end{tabular}

TABLE 2. Minimal normal subgroups of elusive groups of degree 48

With this many elusive groups, and no obvious way to get a compact description, it would seem unlikely that the Polycirculant Conjecture can be proved by first classifying elusive groups.

\section{VERTEX-TRANSITIVE GRAPHS OF ORDER 48}

The class of vertex-transitive graphs plays a central role in algebraic graph theory, often providing extremal cases or illuminating examples in the study of many graphical properties. Although it is not strictly necessary to have a complete list of the transitive groups of degree $d$ in order to compute a complete list of vertex-transitive graphs of order $d$, it is conceptually simple to compute all the vertex-transitive graphs from the transitive groups.

For notational convenience, we say that a graph $\Gamma$ is $G$-vertex-transitive (or just $G$-transitive) if $G \leqslant \operatorname{Aut}(\Gamma)$ and $G$ acts transitively on $V(\Gamma)$. Given a list of all the transitive groups of some fixed degree, in principle it suffices to consider each group $G$ in turn, construct all the $G$ transitive groups, and then merge the lists from the different groups, removing all but one isomorphic copy of each graph.

As stated, this naive algorithm would do far too much work, constructing large numbers of isomorphic copies of most of the graphs. However we can reduce this work in two ways. First we can restrict our attention to the minimal transitive groups, because if $H \leqslant G$ and $H$ is transitive, then any $G$-transitive group is $H$-transitive. Secondly, we can do some work to avoid constructing graphs that are obviously isomorphic to ones that have been, or will be, constructed elsewhere.

The transitive groups of degree 48 and order 48 are necessarily minimal transitive, and we deal with these separately from the larger minimal transitive groups. This separates out the vertex-transitive graphs with a regular subgroup of automorphisms, i.e., the Cayley graphs, from the remainder. This is common practice, because there are a number of interesting questions and conjectures where the distinction between Cayley graphs and non-Cayley graphs appears to be subtle but significant. 
4.1. Cayley graphs. Given a group $G$, and a set of group elements $C \subseteq G$ such that $\mathbf{1}_{G} \notin C$ and $C^{-1}=C$, the Cayley graph $\operatorname{Cay}(G, C)$ is the graph defined as follows:

$$
\begin{aligned}
& V(\operatorname{Cay}(G, C))=G, \\
& E(\operatorname{Cay}(G, C))=\{(g, c g) \mid g \in G, c \in C\} .
\end{aligned}
$$

It is immediate that $G$ is a regular subgroup (acting by right-multiplication) of the automorphism group of $\operatorname{Cay}(G, C)$, and it is well-known that any vertex-transitive graph whose automorphism group has a regular subgroup $R$ is a Cayley graph for $R$. The set $C$ is called the connection set for the Cayley graph and it is precisely the neighbourhood of the vertex $\mathbf{1}_{G}$. (The conditions on $C$ are simply to ensure that the resulting graphs are undirected and loopless.)

While all Cayley graphs are vertex transitive, not all vertex-transitive graphs are Cayley graphs, with the canonical example here being the Petersen graph. For small orders, the vast majority of vertex-transitive graphs are Cayley graphs, but it is not known if this holds in general. In other words, is it true that the proportion of vertex-transitive graphs of order at most $n$ that are Cayley graphs tends to 1 as $n$ increases?

If we define $\Omega=\left\{\left\{g, g^{-1}\right\} \mid g \in G, g \neq \mathbf{1}_{G}\right\}$ then every subset of $\Omega$ determines the connection set for some Cayley graph of $G$ and vice versa. If $G$ has $a$ involutions, and $b$ non-identity element-inverse pairs, then $|\Omega|=a+b$, and so there are exactly $2^{a+b}$ Cayley graphs for $G$. As we are almost always only interested in isomorphism classes of graphs, we need to remove, or preferably never construct, all but one representative of each isomorphism class. There is one obvious source of isomorphisms, namely those arising from the automorphism group of $G$. More precisely, if $\sigma \in \operatorname{Aut}(G)$ then $\operatorname{Cay}(G, C)$ is isomorphic to $\operatorname{Cay}\left(G, C^{\sigma}\right)$. So if we define a Cayley set to be an orbit of $\operatorname{Aut}(G)$ acting on the set of subsets of $\Omega$, then it suffices to consider just one connection set from each Cayley set.

In practice, we fix an arbitrary order on $\Omega$, use GAP to compute the action of $\operatorname{Aut}(G)$ on $\Omega$, and then a simple orderly-style algorithm to compute the lexicographically least representative of each Cayley set. This makes heavy use of Steve Linton's SmallestImageSet [18] to ensure that only lexicographically-least subsets of $\Omega$ are considered at every stage. The theoretical number of Cayley sets for $G$ can be determined by calculating the cycle index polynomial of $\operatorname{Aut}(G)$ acting on $\Omega$ (using the undocumented CycleIndexPolynomial function in Magma) and applying Pólya's Enumeration Theorem. For each of the 52 groups examined, the actual number of Cayley sets constructed by the orderly algorithm matches the theoretical number, giving us a high degree of confidence in this stage of the computation.

Using the list of (representatives of) Cayley sets we next construct the corresponding list of Cayley graphs. Although this list is free of isomorphisms induced by the action of $\operatorname{Aut}(G)$ on the connection sets, there can be additional isomorphisms. Therefore we filter the list of Cayley graphs for each group, removing any graph that is isomorphic to an earlier graph in the list. Sometimes there are no isomorphisms except the ones induced by $\operatorname{Aut}(G)$, and so the final filtering step does not remove any graphs. Groups with this property are called $\mathrm{Cl}$-groups and there is a substantial literature on the still-open question of characterizing $\mathrm{Cl}$-groups. 
There are 52 groups of order 48 and the results of the Cayley graph computations for those groups are given in Table 3. The groups are numbered from 1 to 52 according to their order in the small group libraries of MAGMA and GAP (the groups are in the same order in each library). The group structure is the description returned by the GAP command StructureDescription, the values $a$ and $b$ are the number of involutions and the number of non-identity element-inverse pairs respectively. The column labelled $\mid$ Aut $\mid$ lists the order of the automorphism group of the group. In most cases, the value $2^{a+b} / \mid$ Aut $\mid$ is an approximation (an underestimate) for the number of Cayley sets for that group. Where an entry in the $\mid$ Aut $\mid$ column is marked with an asterisk (such as ${ }^{*} 192$ for group number 8), this indicates a group where $\operatorname{Aut}(G)$ does not act faithfully on $\Omega$. These groups are characterized by the existence of a group automorphism $\sigma \in \operatorname{Aut}(G)$ such that $g^{\sigma} \in\left\{g, g^{-1}\right\}$ for each element $g$. It is known that such a group is either an abelian group (where the inverse map is a group automorphism) or a generalised dicyclic group (Watkins [30]). In each of the cases indicated in Table 3, the kernel of the action of $\operatorname{Aut}(G)$ on $\Omega$ has order 2 , and so these groups yield approximately $2^{a+b+1} / \mid$ Aut| Cayley sets.

The column "Cayley Sets" gives the exact number of Cayley sets obtained from Polya's Enumeration Theorem, while the final column "Cayley Graphs" gives the actual number of pairwise non-isomorphic Cayley graphs. This last step is computationally non-trivial because of the sheer size of some of the lists. For example, there are more than 360 million Cayley graphs for the most prolific group $\left(C_{2} \times D_{24}\right)$.

A group is a CI-group if and only if the number of Cayley graphs is equal to the number of Cayley sets, so the table shows that the abelian group $C_{6} \times C_{2} \times C_{2} \times C_{2}$ is the only CI-group of order 48 .

TABLE 3. Cayley graphs on 48 vertices

\begin{tabular}{ccrrrrr}
\hline No. & Structure & $a$ & $b$ & $\mid$ Aut $\mid$ & Cayley Sets & Cayley Graphs \\
\hline 1 & $C_{3}: C_{16}$ & 1 & 23 & 48 & 496512 & 489376 \\
2 & $C_{48}$ & 1 & 23 & $* 16$ & 2151936 & 2122944 \\
3 & $\left(C_{4} \times C_{4}\right): C_{3}$ & 3 & 22 & 384 & 104224 & 103726 \\
4 & $C_{8} \times S_{3}$ & 7 & 20 & 48 & 3752448 & 3516448 \\
5 & $C_{24}: C_{2}$ & 7 & 20 & 48 & 3561216 & 3337160 \\
6 & $C_{24}: C_{2}$ & 13 & 17 & 96 & 13641984 & 11880240 \\
7 & $D_{48}$ & 25 & 11 & 192 & 364086016 & 360716112 \\
8 & $C_{3}: Q_{16}$ & 1 & 23 & $* 192$ & 275712 & 255696 \\
9 & $C_{2} \times\left(C_{3}: C_{8}\right)$ & 3 & 22 & 96 & 647168 & 597648 \\
10 & $\left(C_{3}: C_{8}\right): C_{2}$ & 3 & 22 & 96 & 586752 & 553168 \\
\hline 11 & $C_{4} \times\left(C_{3}: C_{4}\right)$ & 3 & 22 & 192 & 454176 & 370704 \\
12 & $\left(C_{3}: C_{4}\right): C_{4}$ & 3 & 22 & 96 & 893952 & 611760 \\
13 & $C_{12}: C_{4}$ & 3 & 22 & $* 192$ & 586752 & 484944 \\
14 & $\left(C_{12} \times C_{2}\right): C_{2}$ & 15 & 16 & 96 & 28924416 & 23139848 \\
15 & $\left(C_{3} \times D_{8}\right): C_{2}$ & 17 & 15 & 96 & 47661696 & 45855520 \\
16 & $\left(C_{3}: Q_{8}\right): C_{2}$ & 5 & 21 & 96 & 1102464 & 967024 \\
17 & $\left(C_{3} \times Q_{8}\right): C_{2}$ & 13 & 17 & 96 & 12473472 & 11952272 \\
18 & $C_{3}: Q_{16}$ & 1 & 23 & 96 & 358272 & 308400 \\
19 & $\left(C_{6} \times C_{2}\right): C_{4}$ & 7 & 20 & 192 & 1429024 & 1049296 \\
20 & $C_{12} \times C_{4}$ & 3 & 22 & $* 192$ & 452032 & 431808 \\
\hline 21 & $C_{3} \times\left(\left(C_{4} \times C_{2}\right): C_{2}\right)$ & 7 & 20 & 64 & 3373440 & 2876192 \\
22 & $C_{3} \times\left(C_{4}: C_{4}\right)$ & 3 & 22 & 64 & 1081344 & 904064
\end{tabular}




\begin{tabular}{|c|c|c|c|c|c|c|}
\hline 23 & $C_{24} \times C_{2}$ & 3 & 22 & * 32 & 2336768 & 2183232 \\
\hline 24 & $C_{3} \times\left(C_{8}: C_{2}\right)$ & 3 & 22 & 32 & 1603584 & 1499008 \\
\hline 25 & $C_{3} \times D_{16}$ & 9 & 19 & 64 & 5257920 & 5124112 \\
\hline 26 & $C_{3} \times \mathrm{QD}_{16}$ & 5 & 21 & 32 & 2776320 & 2592224 \\
\hline 27 & $C_{3} \times Q_{16}$ & 1 & 23 & 64 & 447168 & 423232 \\
\hline 28 & $C_{2} \cdot S_{4}=\mathrm{GL}(2,3) \cdot C_{2}$ & 1 & 23 & 48 & 436864 & 431120 \\
\hline 29 & $\operatorname{GL}(2,3)$ & 13 & 17 & 48 & 22758528 & 22589392 \\
\hline 30 & $A_{4}: C_{4}$ & 7 & 20 & 48 & 3343616 & 3230252 \\
\hline 31 & $C_{4} \times A_{4}$ & 7 & 20 & 48 & 3203072 & 3142848 \\
\hline 32 & $C_{2} \times \mathrm{GL}(2,3)$ & 3 & 22 & 48 & 808832 & 791338 \\
\hline 33 & $\left(\left(C_{4} \times C_{2}\right): C_{2}\right): C_{3}$ & 7 & 20 & 48 & 2992000 & 2967742 \\
\hline 34 & $C_{2} \times\left(C_{3}: Q_{8}\right)$ & 3 & 22 & *384 & 386784 & 279616 \\
\hline 35 & $C_{2} \times C_{4} \times S_{3}$ & 15 & 16 & 192 & 16697472 & 14159528 \\
\hline 36 & $C_{2} \times D_{24}$ & 27 & 10 & 384 & 373069248 & 362458536 \\
\hline 37 & $\left(C_{12} \times C_{2}\right): C_{2}$ & 15 & 16 & 96 & 29701632 & 22395608 \\
\hline 38 & $D_{8} \times S_{3}$ & 23 & 12 & 96 & 383280384 & 349815008 \\
\hline 39 & $\left(C_{4} \times S_{3}\right): C_{2}$ & 11 & 18 & 96 & 9219840 & 5452760 \\
\hline 40 & $Q_{8} \times S_{3}$ & 7 & 20 & 288 & 1108224 & 823752 \\
\hline 41 & $\left(C_{4} \times S_{3}\right): C_{2}$ & 19 & 14 & 288 & 34347520 & 30759480 \\
\hline 42 & $C_{2} \times C_{2} \times\left(C_{3}: C_{4}\right)$ & 7 & 20 & *1152 & 428256 & 357264 \\
\hline 43 & $C_{2} \times\left(\left(C_{6} \times C_{2}\right): C_{2}\right)$ & 19 & 14 & 192 & 54719616 & 47641176 \\
\hline 44 & $C_{12} \times C_{2} \times C_{2}$ & 7 & 20 & *384 & 967808 & 806656 \\
\hline 45 & $C_{6} \times D_{8}$ & 11 & 18 & 128 & 6653184 & 5654768 \\
\hline 46 & $C_{6} \times Q_{8}$ & 3 & 22 & 384 & 246912 & 207744 \\
\hline 47 & $C_{3} \times\left(\left(C_{4} \times C_{2}\right): C_{2}\right)$ & 7 & 20 & 96 & 2397184 & 1865216 \\
\hline 48 & $C_{2} \times S_{4}$ & 19 & 14 & 48 & 182656512 & 177923704 \\
\hline 49 & $C_{2} \times C_{2} \times A_{4}$ & 15 & 16 & 144 & 15715936 & 15306700 \\
\hline 50 & $\left(C_{2} \times C_{2} \times C_{2} \times C_{2}\right): C_{3}$ & 15 & 16 & 5760 & 413344 & 411248 \\
\hline 51 & $C_{2} \times C_{2} \times C_{2} \times S_{3}$ & 31 & 8 & 8064 & 72984704 & 71039696 \\
\hline 52 & $C_{6} \times C_{2} \times C_{2} \times C_{2}$ & 15 & 16 & *40320 & 160208 & 160208 \\
\hline
\end{tabular}

The total number of Cayley graphs, after removing isomorphs both within and between the 52 lists of graphs is 1536366616 , approximately 1.54 billion Cayley graphs of order 48 .

4.2. Non-Cayley graphs. The automorphism group of a vertex-transitive graph that is not a Cayley graph is a transitive group that has no regular subgroups. Thus the simplest approach is to consider each minimal transitive group $G$ in turn, compute all the $G$-transitive graphs and then remove both unwanted isomorphs and any Cayley graphs that have accidentally been constructed along the way.

If $G$ is a transitive group then its orbitals are defined to be the orbits of $G$ on $V(G) \times V(G)$. If $\mathcal{O}=(x, y)^{G}$ is an orbital of $G$, then $(y, x)^{G}$ is also an orbital of $G$, called the paired orbital of $\mathcal{O}$. A graph is $G$-transitive if and only if its edge-set is the union of pairs of orbitals of $G$ (identifying an edge $x y$ with a pair of oppositely-directed $\operatorname{arcs}\{(x, y),(y, x)\})$.

An orbital graph of $G$ is a graph whose edge-set is the union of a single pair of orbitals. Let $G^{\prime}$ be the intersection of the automorphism groups of all the orbital graphs of $G$. (This is essentially an undirected version of the 2-closure of a group, sometimes called the strong 2 -closure of the group.) Then any $G$-transitive graph is $G^{\prime}$-transitive and so it is only necessary to process $G^{\prime}$. In itself, this does not reduce the amount of work required because, by definition, $G$ and $G^{\prime}$ have precisely the same pair-closed sets of orbitals to consider. However it is often 
the case that $G^{\prime}$ and $H^{\prime}$ are conjugate even when $G$ and $H$ are not. Therefore by constructing the strong 2-closure of all of the minimal transitive groups and then throwing out all-but-one from each conjugacy class, we end up with a much smaller list of groups to process. This list can be even further reduced by noting that $G^{\prime}$ sometimes has a regular subgroup although $G$, again by definition, does not. In this situation, every $G^{\prime}$-transitive graph is a Cayley graph, and as these have already been constructed, there is no need to process $G^{\prime}$.

The minimal transitive groups of order greater than 48 collectively have 840 pairwise nonconjugate strong 2-closures. It is easy to verify that a small group has no regular subgroups before processing it further, but for the larger groups this becomes too time-consuming. However the larger groups tend to have few orbitals, and so it is easy to construct all of the transitive graphs for these groups. The resulting list of graphs then contains all of the non-Cayley graphs, but also many Cayley graphs that must be removed. Due to the sheer size of the computation, this is a rather lengthy and somewhat intricate process, but on completion we end up with 2501750 non-Cayley graphs, of which 2501630 are connected. Hence the total number of vertex-transitive graphs on 48 vertices is 1538868366 , of which just $0.1625 \%$ are not Cayley graphs.

4.3. Edge-transitive and half-arc transitive graphs. A graph is called edge-transitive if its automorphism group is transitive on edges (i.e., unordered pairs of adjacent vertices) and arc-transitive if it is transitive on its arcs (i.e., ordered pairs of adjacent vertices). An edgetransitive graph might also be vertex-transitive, but there are edge-transitive graphs that are not vertex transitive, in fact some that are not even regular. Conder \& Verret [3] have computed all of the edge-transitive graphs on up to 47 vertices, separately finding those that are vertex transitive, and those that are not.

As a result of the computations reported above, we can go one step further and find the edge-transitive graphs of order 48 that are also vertex-transitive. Thus from the 1.54 billion vertex-transitive graphs of order 48, we extracted 189 edge-transitive graphs, of which 115 are connected, 115 (sic) are twin-free (twins are vertices with the same neighbourhood) and just 71 are both connected and twin-free.

We can also extract a few more interesting graphs from our lists. A graph is called halfarc transitive (or just half-transitive) if it is vertex transitive and edge transitive, but not arc transitive. The most famous, and smallest, such graph is the 4-regular graph on 27 vertices known as the Doyle-Holt graph after its independent discoverers Doyle [6] (originally in an unpublished Masters Thesis at Harvard in 1976) and Holt [10] in 1981.

The data tabulated in Conder \& Verret indicate that there is a single half-arc-transitive graph on 27 vertices (degree 4), 2 on 36 vertices (of degrees 8 and 12), 2 on 39 vertices (degrees 4 and 8 ) and 3 on 40 vertices (all of degree 8). To this we can add another 4 half-arc-transitive graphs on 48 vertices (all of degree 8).

All four of these 8-regular half-arc-transitive graphs are Cayley graphs for at least one group of order 48, with the groups occurring being $\left(C_{4} \times C_{4}\right): C_{3}$ (Group 3 from the list above), $A_{4}: C_{4}$ (group 30), $C_{2} \times C_{2} \times A_{4}$ (group 49) and $\left(C_{2} \times C_{2} \times C_{2} \times C_{2}\right): C_{3}$ (group 50). 


\section{Maximal generating Number of transitive groups of Degree 48}

For an arbitrary group $G$, let $d(G)$ be the minimal size of a generating set of $G$. As we saw earlier, for most of the transitive groups $G$ of degree 48 that are imprimitive with block size 3, the quotient group $\bar{G}:=G / O_{3}(G)$ of $G$ is naturally isomorphic to a transitive group of degree 32 , There are five such groups with $d(\bar{G})=10$, namely TransitiveGroup $(32, i)$ for $i \in\{1422821,1422822,1514676,2424619,2224558\}$, and it turned out that there are also five corresponding groups $G$, also with $d(G)=10$. A lengthy but routine computation showed that these are the only transitive groups of degree 48 with $d(G)>9$.

Among the groups $G$ with minimal block size 2, there are 11 groups with $d(G)=9$, and these have signatures $(2, H)$, where $H=\operatorname{TransitiveGroup}(24, i)$ with $i \in\{9169,21182,23560\}$. The maximum value of $d(G)$ among primitive groups and groups with minimal block size at least 4 is 6 , which arises with block sizes 4 and 6 only.

In [28], the problem of finding numerical upper bounds for $d(G)$ for an arbitrary transitive permutation group $G$ of degree $n$ is considered. It had already been proved in [19] that $d(G)$ is at most $\frac{c n}{\sqrt{\log n}}$ in this case, where $c$ is an unspecified absolute constant. This bound is shown to be asymptotically best possible in [17] (that is, there exists constants $c_{1}, c_{2}$, and an infinite family $\left(G_{n_{i}}\right)_{i=1}^{\infty}$ of transitive groups of degree $n_{i}$, with $c_{1} \leqslant \frac{n_{i}}{d\left(G_{n_{i}}\right) \sqrt{\log n_{i}}} \leqslant c_{2}$ for all $\left.i\right)$.

In [28] it is proved that, apart from a finite list of possible exceptions, the bound $d(G) \leqslant$ $\left\lfloor\frac{c n}{\sqrt{\log n}}\right\rfloor$ holds, where $c:=\frac{\sqrt{3}}{2}$ (and logarithms are to the base 2). This bound is best possible in the sense that $d(G)=\frac{\sqrt{3} n}{2 \sqrt{\log n}}=4$ when $G=D_{8} \circ D_{8}<\mathrm{S}_{8}$ and $n=8$, although it seems likely that there are better bounds that hold for sufficiently large $n$.

The information in the first paragraph above concerning generator numbers in transitive groups of degree 48 has helped the third author to complete the proof of Theorem 1.1 thereby dispensing with the finite list of exceptions. There are, however, a number of other steps in this proof, some of which involve lengthy case-by-case analyses. For this reason, we will just outline the general strategy of the proof in this paper, and the details will be published separately by the third author.

First, by [28, Theorem 5.3], one only needs to prove Theorem 1.1 when $G$ is imprimitive with minimal block size 2 , and $n$ has the form $n=2^{x} 3^{y} 5$ with either $y=0$ and $17 \leqslant x \leqslant 26$; or $y=1$ and $15 \leqslant x \leqslant 35$. Thus, in particular, $G$ may be viewed as a subgroup in a wreath product $2 \imath G^{\Sigma}$, where $\Sigma$ is a set of blocks for $G$ of size 2 . It follows that $d(G) \leqslant d_{G^{\Sigma}}(M)+d\left(G^{\Sigma}\right)$, where $M$ is the intersection of $G$ with the base group of the wreath product, and $d_{G^{\Sigma}}(M)$ is the minimal number of elements required to generate $M$ as a $G^{\Sigma}$-module. With this reduction in mind, the proof of Theorem 1.1 is comprised of two main ingredients: upper bounds on $d_{G^{\Sigma}}(M)$, and upper bounds on $d\left(G^{\Sigma}\right)$. We summarise the third author's approach to these two sub-problems in the next few paragraphs.

We note first that the bulk of the proof is taken up with finding upper bounds on $d_{G^{\Sigma}}(M)$. Since $d_{G^{\Sigma}}(M) \leqslant d_{H}(M)$ for any subgroup $H$ of $G^{\Sigma}$, the strategy of the third author in [28] in this case involved replacing $G^{\Sigma}$ by a convenient subgroup $H$ of $G^{\Sigma}$, and then deriving upper bounds on $d_{H}(M)$, usually in terms of the lengths of the $H$-orbits in $\Sigma$. This approach turns out to be particularly fruitful when $H$ is chosen to be a soluble transitive subgroup of $G^{\Sigma}$, whenever 
such a subgroup exists. When $G^{\Sigma}$ does not contain a soluble transitive subgroup, however, the analysis becomes much more complicated. This led to less sharp bounds, and ultimately, the omitted cases in [28, Theorem 1.1].

The new approach to bounding $d_{G^{\Sigma}}(M)$ involves a careful analysis of the orbit lengths of soluble subgroups in a minimal transitive insoluble subgroup of $G^{\Sigma}$, building on the work in [28] in the case $n=2^{x} 3$. This analysis, together with upper bounds on $d_{H}(M)$ (for soluble $H \leqslant G^{\Sigma}$ ) in terms of the lengths of the $H$-orbits in $\Sigma$, is then used to derive an upper bound for $d_{H}(M)$. An upper bound for $d_{G^{\Sigma}}(M)$ follows.

The second sub-problem is to find an upper bound for $d\left(G^{\Sigma}\right)$. The group $G^{\Sigma}$ is a transitive permutation group of degree $n / 2=2^{x-1} 3^{y} 5$, where $n, x$, and $y$ are as above. The upper bound $d\left(G^{\Sigma}\right) \leqslant \frac{c \frac{n}{2}}{\sqrt{\log \frac{n}{2}}}$ can be derived by using induction on $n$. However, combining this with the upper bounds on $d\left(G^{\Sigma}\right)$ detailed in the previous two paragraphs is not enough to prove Theorem 1.1 in all of the required cases. Therefore, a more careful approach is required. This approach was used in the proof of Lemma 5.12 in the third author's paper [28]. Informally, the idea is as follows. There exists a factorisation $\frac{n}{2}=r_{1} \ldots r_{t}$ of $\frac{n}{2}$ such that either

(1) $d\left(G^{\Sigma}\right) \leqslant \sum_{i}=1^{t-1} d\left(r_{i}, r_{i+1} \ldots r_{t}\right)+\log r_{t}$; or

(2) Either $r_{t} \leqslant 32$, or $r_{t}=48$, and $d\left(G^{\Sigma}\right) \leqslant \sum_{i}=1^{t-1} d\left(r_{i}, r_{i+1} \ldots r_{t}\right)+d_{\text {trans }}\left(r_{t}\right)$.

Here, $d_{\operatorname{trans}}(m):=\max \{d(X): X$ transitive of degree $m\}$. If $2 \leqslant m \leqslant 32$, or if $m=48$, then we know $d_{\text {trans }}(m)$ precisely, by [2], and this paper, respectively.

The function $d(r, s)$ is defined as the maximum of $d_{X}\left(K_{X}(\Delta)\right)$, where

(i) $X$ runs over the transitive permutation groups of degree $r s$ with minimal block size $r$;

(ii) $\Delta$ runs over the blocks for $X$ of size $r$;

(iii) $K_{X}(\Delta)$ is the kernel of the action of $X$ on $\Delta$; and

(iv) $d_{X}\left(K_{X}(\Delta)\right)$ is the minimal number of elements required to generated $K_{X}(\Delta)$ as a normal subgroup of $X$.

Upper bounds on $d(r, s)$ are available from [28]. Thus, we can find upper bounds on $d\left(G^{\Sigma}\right)$ by going through all factorisations of $\frac{n}{2}$, and taking the maximum of the bounds coming from (1) and (2) above. These maximums almost always come from (2). Thus, the new result $d_{\text {trans }}(48)=10$ from this paper plays a vital role in deriving upper bounds on $d\left(G^{\Sigma}\right)$, whence solving the second sub-problem in the proof of Theorem 1.1.

Acknowledgements. We would like to thank Michael Giudici for a number of helpful discussions on transitive permutation groups.

\section{REFERENCES}

[1] Wieb Bosma, John Cannon, and Catherine Playoust. The Magma algebra system. I. The user language. J. Symbolic Comput., 24(3-4):235-265, 1997. Computational algebra and number theory (London, 1993).

[2] John J. Cannon and Derek F. Holt. The transitive permutation groups of degree 32. Experiment. Math., 17(3):307-314, 2008.

[3] Marston D. E. Conder and Gabriel Verret. Edge-transitive graphs of small order and the answer to a 1967 question by Folkman. Algebraic Combinatorics, 2(6):1275-1284, 2019. 
[4] Hannah J. Coutts, Martyn Quick, and Colva M. Roney-Dougal. The primitive permutation groups of degree less than 4096. Comm. Algebra, 39(10):3526-3546, 2011.

[5] Edward Dobson and Joy Morris. Quotients of CI-groups are CI-groups. Graphs Combin., 31(3):547-550, 2015.

[6] Peter G. Doyle. A 27-vertex graph that is vertex-transitive and edge-transitive but not 1-transitive. https://arxiv.org/abs/math/0703861.

[7] Paul Erdös. On a Theorem of Sylvester and Schur. J. London Math. Soc. 9(4):282-288, 1934.

[8] Michael Giudici. New constructions of groups without semiregular subgroups. Comm. Algebra, 35(9):2719$2730,2007$.

[9] Chris Godsil and Gordon Royle. Algebraic Graph Theory, volume 207 of Graduate Texts in Mathematics. Springer-Verlag, New York, 2001.

[10] Derek F. Holt. A graph which is edge transitive but not arc transitive. Journal of Graph Theory, 5(2):201-204, 1981.

[11] Derek Holt and Gordon Royle, A census of small transitive groups and vertex-transitive graphs. J. Symbolic Comput., 101:51-60, 2020.

[12] Alexander Hulpke. Constructing transitive permutation groups. J. Symbolic Comput., 39(1):1-30, 2005.

[13] Peter B. Kleidman. The maximal subgroups of the Steinberg triality groups ${ }^{3} D_{4}(q)$ and of their automorphism groups. J. Algebra, 115(1):182-199, 1988.

[14] Peter B. Kleidman. The maximal subgroups of the Chevalley groups $G_{2}(q)$ with $q$ odd, the Ree groups ${ }^{2} G_{2}(q)$, and their automorphism groups. J. Algebra, 117(1):30-71, 1988.

[15] Peter Kleidman and Martin Liebeck. The subgroup structure of the finite classical groups. London Math. Soc. Lecture Note Ser. 129, Cambridge University Press, 1990.

[16] Mikhail Klin and Dragan Marušič. Problem BCC15.12, Research Problems from the Fifteenth British Combinatorial Conference. Discrete Math, 167-168:605-615, 1997.

[17] L. G. Kovács and M.F. Newman. Generating transitive permutation groups. Quart. J. Math. Oxford Ser. (2) 39(155):361-372, 1988.

[18] Steve Linton. Finding the smallest image of a set. ISSAC 2004, 229-234, ACM, New York, 2004.

[19] Andrea Lucchini. Enumerating transitive finite permutation groups. Bull. London Math. Soc. 30(2):569-577, 1998.

[20] A. Lucchini, F. Menegazzo, and M. Morigi. Asymptotic results for primitive permutation groups and irreducible linear groups. J. Algebra 223(1):154-170, 2000.

[21] Gunter Malle. The maximal subgroups of ${ }^{2} F_{4}\left(q^{2}\right)$. J. Algebra 139(1):52-69, 1991.

[22] Dragan Marušič. On vertex-symmetric digraphs. Discrete Math 36(1):69-81, 1981.

[23] G. A. Miller. List of transitive substitution groups of degree twelve. Quart. Jour. Math., 28:193-231, 1896.

[24] G. A. Miller. The collected works of George Abram Miller, 5 volumes. University of Illinois, 1935-1959.

[25] Gordon F. Royle. The transitive groups of degree twelve. J. Symbolic Comput., 4(2):255-268, 1987.

[26] Gordon F. Royle. An orderly algorithm and some applications in finite geometry. Discrete Math., 185(13):105-115, 1998.

[27] Michio Suzuki. On a class of doubly transitive groups. Ann. of Math. 75(2):105-145, 1962.

[28] Gareth M. Tracey. Minimal generation of transitive permutation groups. J. Algebra 509:40-100, 2018.

[29] Gareth M. Tracey. Upper bounds on the minimal number of elements required to generate a transitive permutation group. Submited to J. Algebra.

[30] Mark E. Watkins, On the Action of Non-Abelian Groups on Graphs. J. Comb. Theory Ser. B 11:95-104, 1971.

[31] Robert Wilson et al. Atlas of Finite Group Representations, http://brauer.maths.qmul.ac.uk/Atlas/v3/. 
Mathematics Institute, University of WARWick

Email address: D.F.Holt@warwick.ac.uk

Mathematics and Statistics, University of Western Australia

Email address: gordon.royle@uwa.edu.au

Mathematical Institute, University of Oxford

Email address: garethtracey1@gmail.com 